Int. J. Electrochem. Sci., 14 (2019) $7884-7898$

International Journal of

ELECTROCHEMICAL

SCIENCE

www.electrochemsci.org

\title{
Medium Effect on the Electropolymerization and Electro-optical Properties of PEDOS
}

\author{
Kai Qu ${ }^{\S 1}$, Hongtao Li ${ }^{\S 1}$, Nannan Jian ${ }^{1}$, Baoyang Lu ${ }^{2, *}$, Ximei Liu ${ }^{2}$, Jingkun Xu ${ }^{1,3, *}$ \\ ${ }^{1}$ School of Chemistry \& Chemical Engineering, Jiangxi Science \& Technology Normal University, \\ Nanchang 330013, Jiangxi, China \\ ${ }^{2}$ School of Pharmacy, Jiangxi Science \& Technology Normal University, Nanchang 330013, Jiangxi, \\ China \\ ${ }^{3}$ School of Chemistry and Molecular Engineering, Qingdao University of Science and Technology, \\ Qingdao 266042, Shandong, China \\ *E-mail: 1by1258@163.com and xujingkun1971@yeah.net \\ $\S$ These authors contributed equally to this work.
}

doi: $10.20964 / 2019.08 .74$

Received: 19 March 2019 / Accepted: 6 June 2019 / Published: 30 June 2019

3,4-Ethylenedioxyselenophene (EDOS), an important derivative of 3,4-ethylenedioxythiophene (EDOT), has better optoelectronic properties including lower band gap, better interchain charge transfer, lower oxidation and reduction potentials. In this work, the electropolymerization behavior of EDOS, and the electrochemistry, surface morphology, optical and electrochromic properties of the resultant PEDOS films are comparatively investigated in different solvent-electrolyte systems $\left(\mathrm{CH}_{2} \mathrm{Cl}_{2}-\mathrm{Bu}_{4} \mathrm{NPF}_{6}, \mathrm{CH}_{2} \mathrm{Cl}_{2}-\mathrm{BmimPF}_{6}\right.$, and pure $\left.\mathrm{BmimPF}_{6}\right)$. We find that solvent-electrolyte systems exert influence on the electropolymerization behavior of EDOS monomer. EDOS displays lower onset oxidation potentials in $\mathrm{CH}_{2} \mathrm{Cl}_{2}-\mathrm{BmimPF}_{6}$ and pure $\mathrm{BmimPF}_{6}$ than commonly employed $\mathrm{CH}_{2} \mathrm{Cl}_{2}-$ $\mathrm{Bu}_{4} \mathrm{NPF}_{6}$. PEDOS can be facilely electrodeposited in all these systems. Electrochromic results suggest that PEDOS film resulted from $\mathrm{CH}_{2} \mathrm{Cl}_{2}-\mathrm{Bu}_{4} \mathrm{NPF}_{6}$ medium exhibit relatively good transmittance changes $(\triangle \mathrm{T} \%)$ of $26.5 \%$, high coloration efficiency of $80 \mathrm{~cm}^{2} \mathrm{C}^{-1}$. However, the addition of $\mathrm{BmimPF}_{6}$ reveal a compact morphology, and enhanced the electrochemical stability and open circuit memory for PEDOS film. From these results, PEDOS can be further explored as electrochromic materials towards indoor electrochromic products and flexible displays.

Keywords: Conducting polymers, Electropolymerization, Electrochromics, 3,4Ethylenedioxyselenophene, Medium Effect

FULL TEXT 
(C) 2019 The Authors. Published by ESG (www.electrochemsci.org). This article is an open access article distributed under the terms and conditions of the Creative Commons Attribution license (http://creativecommons.org/licenses/by/4.0/). 